\title{
Astrocytic-neuronal crosstalk gets jammed: Alternative perspectives on the onset of neuropsychiatric disorders
}

\author{
Celia Roman $^{1}$ | Luisa Egert $^{1}$ | Barbara Di Benedetto ${ }^{1,2}$ (D)
}

${ }^{1}$ Department of Psychiatry and Psychotherapy, University of Regensburg, Regensburg, Germany

${ }^{2}$ Regensburg Center of Neuroscience, University of Regensburg, Regensburg, Germany

\section{Correspondence}

Barbara Di Benedetto, Department of Psychiatry and Psychotherapy, University of Regensburg, Franz-Josef-Strauß Allee 11, 93053 Regensburg, Germany.

Email: Barbara.Di-Benedetto@ukr.de

\section{Funding information}

Deutsche Forschungsgemeinschaft, Grant/ Award Number: GRK2174 ; Department of Psychiatry and Psychotherapy, University of Regensburg

\begin{abstract}
Investigating interactions of glia cells and synapses during development and in adulthood is the focus of several research programmes which aim at understanding the neurobiology of brain physiological and pathological processes. Both glia-specific released and membrane-bound proteins play essential roles in the development, maintenance and functionality of synaptic connections. Alterations in synaptic contacts in specific brain areas are hallmarks of several brain diseases, such as major depressive disorder, autism spectrum disorder and schizophrenia. Thus, a deeper knowledge about putative astrocyte dysfunctions which might affect the synaptic compartment is warranted to improve treatment options. Here, we present the latest advances about the role of glia cells in orchestrating the arrangement of synapses and neuronal networks in physiological and pathological states. We specifically focus on the role of astrocytes in the phagocytosis of neuronal synapses as a novel mechanism which drives the refinement of neuronal circuits and might be affected in pathological conditions. Finally, we propose this astrocyte-dependent mechanism as a putative alternative target of pharmacological interventions for the treatment of brain disorders.
\end{abstract}

\section{K E Y W O R D S}

astrocytes, neuropsychiatric disorders, pruning, synapse remodelling

\section{1 | INTRODUCTION}

Among all glia cells, astrocytes represent a highly heterogeneous class of neural cells of neuroectodermal origin. They can be classified into two major groups: protoplasmic astrocytes, whose processes ensheath synapses and blood vessels in the grey matter, and fibrous astrocytes, which contact nodes of Ranvier and blood vessels in the white matter (for a review, see Tabata, 2015). The revolutionary realization that astrocytes are not only necessary for neuronal support, but

\footnotetext{
Abbreviations: ABCA1, ATP-binding cassette subfamily A; AMPAR, $\alpha$-amino-3-hydroxy-5-methyl-4-isoxazolepropionic acid receptor; AQP4, aquaporin 4; ASDs, autism spectrum disorders; BDNF, brain-derived neurotrophic factor; CNS, central nervous system; CUS, chronic unpredictable stress; Cx, connexin; DAAO, D-amino acid oxidase; E/I, excitation/inhibition; GABA, $\gamma$-aminobutyric acid; GAD, glutamic acid decarboxylase; GULP1, engulfment adaptor PTB domain containing 1; GWAS, genome-wide association study; iPSCs, induced pluripotent stem cells; LGN, lateral geniculate nucleus; LTP, long-term potentiation; MDD, major depressive disorder; MEGF10, Multiple EGF Like Domains 10; MERTK, proto-oncogene tyrosine-protein kinase MER; NMDAR, N-methyl-D-aspartate receptor; PFC, prefrontal cortex; PS, phosphatidylserine; PSD-95, postsynaptic density protein 95; RGCs, retinal ganglion cells; SCZ, schizophrenia; SR, serine racemase; SV2A, synaptic vesicle glycoprotein 2A.

Edited by: Ania Majewska

This is an open access article under the terms of the Creative Commons Attribution-NonCommercial License, which permits use, distribution and reproduction in any medium, provided the original work is properly cited and is not used for commercial purposes.

(ㅇ) 2020 The Authors. European Journal of Neuroscience published by Federation of European Neuroscience Societies and John Wiley \& Sons Ltd.
} 
actively contribute to synaptic formation and function, led to an increased scientific interest in these cells (for review see Perea, Navarrete, \& Araque, 2009).

The bidirectional communication between astrocytes and neurons which modulates synaptic structure and plasticity is enabled by the close spatial association between the peripheral astrocyte processes and neuronal synaptic contacts to form the so-called "tripartite synapse" (Araque, Parpura, Sanzgiri, \& Haydon, 1999; Perea et al., 2009). Synapses are highly dynamic structures, which can be rapidly modified upon neuronal activity. Dendritic spines undergo activity-dependent structural remodelling as a result of experience-dependent plasticity events, especially important for the induction and maintenance of long-term potentiation (LTP), the cellular correlate of learning and memory (Murakoshi \& Yasuda, 2012). The importance of a balanced synaptic activity becomes evident when considering that synaptic impairments have been ascribed to various psychiatric pathologies. Astrocytes actively influence synaptic properties through an array of secreted and contact-dependent signals. Therefore, the hypothesis that an altered astrocytic-neuronal crosstalk may contribute to synaptic malfunctions and thereby trigger the onset of brain disorders is gaining an increasing biomedical relevance (Chung, Welsh, Barres, \& Stevens, 2015; Di Benedetto, Rupprecht, \& Czéh, 2013). Furthermore, the targeting of molecules and pathways by which astrocytes influence synapses may represent an alternative and/or complementary therapeutic approach to improve the treatment of psychiatric disorders (Czéh \& Di Benedetto, 2013; Di Benedetto et al., 2013).

Here, we review the main mechanisms used by astrocytes to modulate synaptic formation and functions. Furthermore, we discuss in more details some of those mechanisms and how they relate to psychiatric disorders, with particular emphasis on depression, autism spectrum disorder and schizophrenia. Finally, we propose alternative paths which may be relevant for the treatment of such disorders.

\section{INFLUENCE OF ASTROCYTIC- RELEASED MOLECULES ON SYNAPTIC REMODELLING}

The first evidence which suggested that glia cells, and specifically astrocytes, may influence synaptic formation/degradation/function was based on the observation that astrocytes and most of the synapses simultaneously mature during the early stages of postnatal brain development, a very delicate time span also known as "sensitive" or "critical" period. This dynamic interval of synapse development and refinement in the postnatal brain under the control of glia cells is crucial for a physiological neuronal circuit formation (Farhy-Tselnicker \& Allen, 2018).
Initial in vitro experiments of cultured neurons grown either in direct contact with astrocytes or with astrocyte conditioned media revealed a remarkable increase in synaptic numbers and activity with respect to neurons grown alone. These results suggested an essential role of both contact- and soluble factors-mediated effects for a proper synapse formation and maturation (Allen et al., 2012; Barker \& Ullian, 2008). Several early studies suggest that alterations in the contribution of these mechanisms to synaptogenesis and subsequent activation of different intracellular signalling pathways vary across neuronal populations (Barker, Koch, Reed, Barres, \& Ullian, 2008; Hama, Hara, Yamaguchi, \& Miyawaki, 2004; Mauch et al., 2001; Ullian, Sapperstein, Christopherson, Barres, \& Barde, 2001). A recent excellent example of an astrocyte contact-mediated synaptogenic mechanism is represented by the interaction between the astrocytic neuroligin and neuronal neurexin during astrocyteneuron adhesion processes, which leads to a local regulation of synapse development (Singh et al., 2016; Stogsdill et al., 2017).

Beyond contact-mediated mechanisms, the synchronization of astrocyte and synapse development, which supports the successful regulation of postnatal neuronal network refinement, is additionally facilitated by a tightly controlled chronological expression of various soluble synaptogenic factors. Astrocytes control excitatory synapse formation/ function in three major ways described so far (reviewed in Barker \& Ullian, 2008; Chung, Allen, \& Eroglu, 2015). First, astrocytic thrombospondins increase the number of synapses, which exhibit a normal morphology, but are silent, since they express $N$-methyl-D-aspartate receptors (NMDAR), but lack $\alpha$-amino-3-hydroxy-5-methyl-4-isoxazolepropionic acid receptors (AMPAR) on postsynaptic membranes (Clarke \& Barres, 2013; Crawford, Jiang, Taylor, \& Mennerick, 2012; Kucukdereli et al., 2011; Stogsdill \& Eroglu, 2017). Second, postsynaptic functionality may be achieved by Glypican4and 6-regulated AMPAR localization and clustering to the postsynaptic membrane (Allen et al., 2012). Third, astrocytes increase the probability of transmitter release at presynaptic boutons, by cholesterol secretion (Goritz, Mauch, \& Pfrieger, 2005; Mauch et al., 2001).

In contrast to excitatory synapse development, the astrocytic influence on inhibitory synapses is still widely unexplored. However, a strong influence of soluble factors released by astrocytes, such as brain-derived neurotrophic factor, is suggested to influence inhibitory synapse formation (Elmariah, Hughes, Oh, \& Balice-Gordon, 2004; Hughes, Elmariah, \& Balice-Gordon, 2010).

Astrocytes are additionally dynamic partners of neurons to support brain synaptic communication. NMDAR and AMPAR are among the key players in excitatory neurotransmission and synaptic plasticity. The activation of NMDAR requires the binding of both glutamate and its co-agonist 
D-serine, a soluble molecule which has been considered to be almost exclusively released by astrocytes (Panatier et al., 2006). More recently, this view has been questioned after few studies have shown that neurons might also represent a source of D-serine in physiological conditions, whereas reactive astrocytes may become the main source under pathological conditions (Ivanov \& Mothet, 2018). D-serine controls both NMDAR activity and synaptic memory (Henneberger, Papouin, Oliet, \& Rusakov, 2010). In particular, a proper D-serine regulation is essential for a balanced NMDARmediated synaptic transmission in the developing brain, thereby promoting synapse maturation and axonal stabilization (Van Horn, Strasser, Miraucourt, Pollegioni, \& Ruthazer, 2017). In fact, selective loss of function of D-serine reduces synaptic events and prevents induction and expression of NMDAR-dependent LTP in the visual cortex (Meunier et al., 2016). In addition, worth to be repeated, the localization and clustering of AMPAR to the postsynaptic membrane, essential to lead the switch from a "silent" to an "active" synaptic state, are induced by Glypican $4 / 6$ released by astrocyte (Allen et al., 2012).

\section{3 | INFLUENCE OF ASTROCYTIC MEMBRANE-BOUND MOLECULES ON SYNAPTIC REMODELLING}

Among others, one critical mechanism for the regulation of neuronal interconnectivity is the synaptic pruning mediated by glia cells, specifically astrocytes and microglia (Chung et al., 2013; Stevens et al., 2007). This crucial mechanism allows flexible and precise adaptations of neuronal circuits to endogenous or environmental stimuli through an extensive activity-dependent synaptic remodelling. Such a neuronal circuit refinement is controlled by astrocytes through synaptic pruning in two major ways, one more "passive" and one more "active." First, astrocyte may "passively" support synapse elimination by microglia, via induction of the complement component $\mathrm{C} 1 \mathrm{q}$ expression, which represents a starting component of the classical complement cascade. This process exhibits its strongest activity in the first two postnatal weeks and is hypothesized to be regulated by astrocyte-derived soluble factors (Bialas \& Stevens, 2013; Luchena, Zuazo-Ibarra, Alberdi, Matute, \& Capetillo-Zarate, 2018; Stevens et al., 2007). Second, astrocytes are able to "actively" engulf synapses in a strongly activity-dependent manner. Interestingly, this phagocytic activity of astrocytes is not only present during brain development, but persists into adulthood (Chung et al., 2013; Lee \& Chung, 2019).

The idea that astrocytes are able to actively prune synapses was proposed after a gene expression study which compared gene activity among astrocytes, neurons and oligodendrocytes. Cahoy et al. found specifically in astrocytes an upregulation of a set of genes that have been implicated in phagocytosis, suggesting that these cells may act as professional phagocytes (Cahoy et al., 2008). Among them, especially two encode for transmembrane receptors which mediate synapse or debris engulfment, the Multiple EGF Like Domains 10 (MEGF10) and the proto-oncogene tyrosine-protein kinase MER (MERTK), (Chung et al., 2013). Remarkably, these receptors play a role in a phagocytic pathway evolutionarily conserved in several species. MEGF10 is an orthologue of the Drosophila melanogaster Draper and of the Caenorhabditis elegans CED-1 (Fullard \& Baker, 2015; Mapes et al., 2012; Ziegenfuss et al., 2008), whereas MERTK is a member of the MER/AXL/TYRO3 receptor kinase family. Along with the integrin pathway, MERTK regulates CrKII/DOCK180/Rac1 in order to control the rearrangement of the actin cytoskeleton during the phagocytic process (Mao $\&$ Finnemann, 2015). The MEGF10 pathway initiates phagocytosis with the mediation of the protein GULP, engulfment adaptor PTB domain containing 1 (GULP1). Additionally, the ATP-binding cassette subfamily A member 1 (ABCA1) is also required to favour phagocytosis, but its function downstream to MEGF10 is still unclear (Hamon et al., 2006). These receptors have similar roles in different species. In the fly Drosophila, Draper is upregulated in glial cells after axonal injury and drives the clearance of axonal debris. In draper mutants, glia cells fail to expand their membranes and engulf injured axons (MacDonald et al., 2006). In the worm Caenorhabditis, CED1 is localized on the surface of phagocytic cells and is able to recognize cell corpses when clustering together around targeted apoptotic cells (Zhou, Hartwieg, \& Horvitz, 2001). In mammals, the MEGF10 and MERTK pathways are mostly activated during development and specifically mediates synaptic pruning (Chung et al., 2013). These data indicate that astrocytes are important effectors of synaptic pruning during development and in adulthood in an evolutionarily conserved way.

Alterations in the communication between microglial cells and astrocytes may also influence synapse loss in brain diseases, a topic which has also been discussed in a recent review by Wilton and colleagues (Wilton, Dissing-Olesen, \& Stevens, 2019). In brief, both cell types control synapse elimination through the phagocytosis of synaptic structures in an activity-dependent manner and reshape in this way the connectivity of neuronal circuits (Chung et al., 2013; Schafer et al., 2012). The mostly studied mechanism used by both microglial cells and astrocytes to mediate synapse elimination in the developing central nervous system (CNS) is the activation of the classical complement pathway (Chu et al., 2010; Schafer et al., 2012; Stevens et al., 2007). MEGF10 phagocytic receptor binds with high affinity to C1q (Iram et al., 2016), which further recognizes and binds phosphatidylserine (PS) expressed on the surface of apoptotic cells, thereby acting as a "bridging" molecule between interacting 
cells (Païdassi et al., 2008). PS represents one of the so-called "eat-me" signals expressed on synapses, which is then recognized by MEGF10 and MERTK receptors localized on astrocytes, thus serving as a tag to label synapses that have to be eliminated. It has been observed that the engulfment ability of astrocytes is also highly dependent on the presence of protein $\mathrm{S}$ in blood serum, the latter representing the factor responsible for serum-stimulated phagocytosis (Anderson et al., 2003; Chung et al., 2013).

To study the process of synapse elimination, the visual system has been widely used due to its well-characterized experience-dependent synaptic refinement (Wiesel \& Hubel, 1963). In this system, during development, axonal projections from retinal ganglion cells (RGCs) form supernumerary synapses with neurons in the dorsal lateral geniculate nucleus (LGN) of the thalamus. Single LGN neuron receives RGC inputs from a single eye during the first postnatal week. Subsequently, upon eye opening and increased neuronal activity, some RGC inputs onto each LGN neuron are removed and the remaining ones get stabilized. Astrocytes play a critical role in this removal of excessive synapses through phagocytosis to favour the reshaping of the visual circuit. Mice lacking MEGF10 and MERTK phagocytic receptors since developmental stages displayed about 50\% reduction in their engulfment ability. Thus, they fail in the refinement of thalamic synaptic connections and display an excess of functional synapses. Using this model, it has been shown that synapse engulfment and elimination by astrocytes persist in the adult CNS (Chung et al., 2013).

The highest rate of synaptic pruning, however, occurs during development, while afterwards it decreases with age. Synapse formation and maturation occur in parallel with astrogenesis and astrocyte maturation during the so-called "critical period," the time of postnatal development during which most of neuronal circuit refinement takes place (Chung, Welsh, et al., 2015). Although neurons send out projections before birth, synapses only begin to form and get stabilized from the first week of postnatal development, concurrently with the appearance of higher numbers of astrocytes (FarhyTselnicker \& Allen, 2018). This may be one of the most important periods in terms of plastic changes, with a higher rate of phagocytosis of weak synapses to favour the selective stabilization of the stronger ones in adulthood. Moreover, because of the lower rates of neurogenesis in adulthood (Snyder, 2019), with consequent reduced replacement of neurons throughout the brain, the reshaping of circuits seems to be less relevant than during development.

In addition to the regulation of synaptic morphology, several astrocyte-specific membrane-bound proteins are involved in the control of synaptic transmission through the modulation of ion concentrations in the synaptic cleft (Voglis \& Tavernarakis, 2006). To mention a few, aquaporin 4 (AQP4), a transmembrane protein which acts as water channel and is selectively expressed on the endfeet of astrocyte processes, connexins 30 and 43 (Cx30 and Cx43), the transmembrane proteins that form gap junctions, and the excitatory amino acid transporters EAAT1 (known as GLAST in rodents) and EAAT2 (GLT-1) have been associated with a direct or indirect modulation of $\mathrm{K}^{+}$and/or $\mathrm{Ca}^{2+}$, glutamate and D-serine availability, which are necessary to regulate both basal and activity-dependent synaptic neurotransmission (Mayorquin, Rodriguez, Sutachan, \& Albarracín, 2018; Strohschein et al., 2011).

\section{4 | ROLE OF ASTROCYTE- MEDIATED SYNAPSE REMODELLING IN PSYCHIATRIC DISORDERS}

\section{1 | Major depressive disorder}

Major depressive disorder (MDD) is characterized by core symptoms of depressed mood or dysphoria. Other symptoms or behaviours present in depressive patients include loss of interest in daily activities, changes in sleep patterns and appetite, sense of guilt and hopelessness, fatigue, restlessness, concentration problems and suicidal thoughts (Otte et al., 2016). According to the World Health Organization, the number of people suffering from MDD has increased in the last decade. Nowadays, MDD is among the leading causes of disability worldwide and is a major contributor to the overall global burden of disease (DALY, World Health Organization, 2017; https://www.who.int/health-topics). The role of the serotonin and noradrenaline neurotransmitter systems in mental illnesses including MDD is well recognized. Furthermore, there are evidences showing that abnormalities in glial cells may alter normal brain function and likely contribute to the development of mood disorders (Di Benedetto \& Rupprecht, 2013; Rajkowska, Hughes, Stockmeier, Javier Miguel-Hidalgo, \& Maciag, 2013; Schmidtner et al., 2019; Banasr, Dwyer, \& Duman, 2011). Specifically, modifications in astrocyte morphology, numbers and related markers-that is glial fibrillary acidic protein, calcium-binding protein S100 $\beta, \mathrm{Cx}, \mathrm{AQP} 4$, EAAT1/2-in relevant limbic brain structures are key features in the pathology of MDD, as observed in postmortem histopathological studies (Rajkowska \& Stockmeier, 2013). Additionally, morphological studies made after repeated stress paradigms in rodents, used to induce depressive-like behaviours, showed the induction of changes in volume of hippocampus and prefrontal cortex (PFC), with associated reduction in size, or loss of, glial cells and neurons in these regions (Duman \& $\mathrm{Li}$, 2012; Liu \& Aghajanian, 2008). In particular, both repeated restraint stress and chronic immobilization stress in rats produced dendritic atrophy in pyramidal neurons of the medial PFC and in the CA3 area of the hippocampus, with decreased 
apical dendritic branch length and spine density in distal branches (Christian, Miracle, Wellman, \& Nakazawa, 2011; Liu \& Aghajanian, 2008; Radley et al., 2006). Remodelling of hippocampal spine synapses has also been analysed at the ultrastructural level. Electron microscopy studies showed that inescapable footshock or injection of the stress-inducing steroid corticosterone caused an acute and persistent loss of spine synapses in the rat hippocampus, while the antidepressant (AD) desipramine (DMI) reversed both the structural and behavioural deficits (Hajszan et al., 2009). Other multimodal stressors also provoked a robust loss of postsynaptic density protein 95 (PSD-95)-positive puncta in hippocampal areas, affecting learning and memory (Maras et al., 2014). Rats subjected to long-term stress further showed a reduction in the number of axo-spinous synapses in deep layers of the medial PFC, suggesting an impaired network connectivity among cortical neurons (Csabai, Wiborg, \& Czéh, 2018) and confirming previous observations on a loss or reduction of total dendritic arbours in the PFC (Liu \& Aghajanian, 2008; Radley et al., 2006). In support of the above-mentioned changes, in vivo evidences have linked the lower synaptic density to network alterations and symptoms of depression by analysing the synaptic vesicle glycoprotein $2 \mathrm{~A}$ (SV2A) as an indirect estimator of synaptic density. Individuals with MDD showed lower SV2A density compared to healthy controls (Holmes et al., 2019). Among the hypothesis proposed for depression, a recent one suggests an altered functioning of excitatory synapses to explain the excessive concentration of glutamate measured in parenchymal brain tissues of both animal models of depression and postmortem human brain tissues of MDD patients. More specifically, chronic stress and an individual genetic susceptibility to develop MDD may cause changes in the strength of subsets of glutamatergic synapses in multiple brain areas, which might induce changes in glutamate neurotransmission and lead to several dysfunctions, such as the one in the cortico-mesolimbic reward circuitry which underlies many symptoms of depression (Duman, Aghajanian, Sanacora, \& Krystal, 2016; Popoli, Yan, McEwen, \& Sanacora, 2012; Thompson et al., 2015) .

Considering the previously described highly relevant role of astrocytes in the regulation of synaptic numbers, morphology and functions, their examination in animals exposed to various stressors has, not surprisingly, revealed significant changes in astrocyte-specific proteins which impact such parameters. Specifically, acute stress and chronic unpredictable stress (CUS) decrease the diffusion of a special channel-permeable dye through astrocytes, with a corresponding reduced expression of the gap junction forming proteins $\mathrm{Cx} 30$ and Cx43, respectively (Sun, Liu, Yuan, Li, \& Chen 2012; Murphy-Royal et al. 2020). In line with these data, a decrease in $\mathrm{Cx} 43$ occurs in the dorsolateral PFC of people who committed suicide and in the locus coeruleus and hippocampus of subjects diagnosed with MDD (Bernard et al., 2011; Ernst et al., 2011; Medina et al., 2016). Furthermore, mice lacking AQP4, which display an impaired $\mathrm{K}^{+}$buffering capacity with a putatively consequent hampered synaptic transmission, showed remarkable signs of enhanced depressive-like behaviours, demonstrated by longer immobility times in the forced swimming and tail suspension tests (Kong et al., 2014). In line with this, the coverage of blood vessels by the AQP4-positive endfeet of astrocytes is reduced by $50 \%$ in MDD patients and in an animal model of depression, suggesting that a lowered expression or a mislocalization of AQP4 may further underlie the pathogenesis of MDD (Di Benedetto et al., 2016; Rajkowska et al., 2013). A reduced expression of the astrocyte-specific glutamate transporters EAAT1 and EAAT2 has also been described in the anterior cingulate cortex, dorsolateral PFC, orbitofrontal cortex, in locus coeruleus and hippocampus of MDD patients, thus suggesting an additional involvement of dysfunctional astrocytes in the previously mentioned "glutamatergic hypothesis of depression" (Bernard et al., 2011; Choudary et al., 2005; Medina et al., 2016; Miguel-Hidalgo et al., 2010).

All cellular changes described so far indicate that both neurons and astrocytes display several abnormal characteristics in mood disorders. It is therefore conceivable that such modifications affect both the single cell types, as well as the astrocyte-neuron communication, which may influence the formation, elimination and functions of synapses. The remodelling of synapses is a plasticity event, which has key roles during development and specifically influences learning and memory processes, which are severely impaired in MDD. In a previous work, our group has examined the effect of the $\mathrm{AD}$ DMI on hippocampal LTP in brain slices from male CD-1 mice. We first showed that LTP induction selectively activated the ERK/MAPK in astrocytes of the stratum radiatum of the hippocampal CA1 region, but not in CA1 neurons. We further demonstrated that DMI acutely inhibited LTP through a cell-type-specific dampening of ERK activation in astrocytes, which consequently hindered synaptic potentiation. In particular, our results indicated the enhancement of the glianeuron bidirectional ephrinA3/EphA4 signalling pathway as a novel pharmacological downstream target of DMI (Tanasic et al., 2016).

In addition to synaptic plasticity mechanisms, astrocyte-mediated phagocytosis may play a critical role in reshaping neuronal circuits. If the proper pruning of synapses does not take place at specific time points (such as during the critical period), an improper remodelling of circuits may occur and this can evolve into the onset of mood disorders in adulthood. To examine this aspect, our group is currently evaluating whether ADs may activate MEGF10 receptor to remodel glutamatergic synapses and thereby restore disrupted neuronal circuits in an animal model of MDD through phagocytosis. Preliminary data suggest that ADs induce an 
astrocyte-mediated early synaptic pruning in the PFC of adult rats, suggesting that these drugs may reactivate a cellular programme similar to the one which drives postnatal brain neuronal circuit formation, when MEGF10 is highly expressed in astrocytes. Thus, astrocytes may additionally represent $\mathrm{AD}$ targets and play an essential role in synaptic network reshaping upon drug treatments.

\section{2 | Autism spectrum disorders}

Autism spectrum disorders (ASDs) are neurodevelopmental disorders characterized by varying degrees of impaired social interaction and communication. Several evidences indicate that autism-related mutations disrupt genes which are regulated by, and regulate, neuronal activity and synaptic formation and/or function (Emberti Gialloreti \& Curatolo, 2018). Although the molecular mechanisms of ASDs are still poorly understood, several hypotheses point to an abnormal synapse development as an underlying putative cause of some forms of ASD. Indeed, some features of the disease have been ascribed to a misbalanced ratio of excitatory-inhibitory synapses (Rubenstein \& Merzenich, 2003). Moreover, alterations in glutamate concentrations have also been detected in the pregenual anterior cingulate cortex of pedriatic ASD patients (Bejjani et al., 2012). In support of the latter observation, transgenic animal models have been generated to triplicate the gene dosage of Ube $3 a$, a protein ligase expressed in mature neurons and affected in ASD patients. These animals show a subset of behaviours typical of ASD, such as increased repetitive behaviour and impaired social interaction and communication. When analysed at the microscopical level, these animals displayed a suppressed glutamatergic synaptic transmission as a result of modified presynaptic release probability, with consequent changes in synaptic glutamate concentration and postsynaptic action potential coupling (Smith et al., 2011). Because of the role that astrocytes play in the maintenance of appropriate levels of glutamate in the synaptic cleft, the glutamine synthetase (GS), an enzyme expressed in astrocytes with a critical role in glutamate synthesis, has been examined and found to be increased in the blood plasma of autistic patients (Hamed et al., 2018). Furthermore, other astrocyte-specific proteins such as AQP4 and Cx43 are altered in postmortem brains of ASD patients, thereby suggesting an underlying abnormal astrocyte-neuron communication in the pathophysiology of ASD. In fact, changes in the expression of these proteins may reflect disruptions in blood-brain barrier functionality and cell-to-cell communication (Fatemi, Folsom, Reutiman, \& Lee, 2008).

Some years ago it was suggested that ASD and related disorders might depend on a deregulated ratio between excitatory and inhibitory neurotransmission, leading to a hyperexcitability of cortical circuits (Rubenstein \& Merzenich, 2003). At the neuronal level, the excitation/ inhibition (E/I) balance in the cortex is controlled by the activity of glutamatergic and $\gamma$-aminobutyric acid (GABA) ergic neurons. In the cortex, excitatory glutamatergic neurons outnumber inhibitory GABAergic neurons by about four to one. It has been proposed that an imbalanced E/I may be involved in the pathogenesis of this disorder as a consequence of several mutations in genes coding for various components of these two neurotransmitter systems in combination with environmental variables (Rubenstein \& Merzenich, 2003).

Identification of diseased phenotypes and the use of appropriate functional assays are essential in understanding psychiatric diseases. Among the methods developed for this aim, the generation of different subtypes of cells starting from patient-derived induced pluripotent stem cells (iPSCs) has gained a lot of attention in the biomedical community. Several groups have generated human astrocytes from iPSCs of psychiatric patients and found that, for example, ASD-derived astrocytes interfered with proper neuronal development when grown together with primary neurons in co-culture in vitro models. Specifically, ASD-derived neurons show a severely impaired neuronal morphology with decreased synaptogenesis, when grown with ASD-derived astrocytes. However, co-culturing with control-derived astrocytes rescued this phenotype (Russo et al., 2018). These data suggest that also for ASD, astrocyte-specific molecules may fail to properly execute their functions and lead to the formation of aberrant neuronal synaptic contacts. Some of them have been already mentioned (AQP4 and Cx30/43), although the exact molecular mechanisms beyond their effects are still a matter of currently ongoing studies. Among alternative unexplored mechanisms, which could also lead to an E/I imbalance, glia-mediated phagocytosis is worth to be mentioned here. In fact, the preferential astrocyte-mediated phagocytic activity towards glutamatergic synapses (Chung et al., 2013) may indeed cause a dysbalanced development of the excitatory-to-inhibitory synaptic density ratio, with consequent excess of excitatory synapses due to a lack of MEGF10 or MERTK functionality during developmental stages.

\section{3 | Schizophrenia}

Schizophrenia (SCZ) is a debilitating mental illness that impairs mental functions and social interactions and affects about $1 \%$ of the population. Most common symptoms of SCZ are hallucination, delusions, loss of pleasure and will and social withdrawal. Schizophrenic people additionally present cognitive disorders with impaired executive functions, memory and speed of mental processing (Marder \& Cannon, 2019). Both genetic and environmental factors may be involved in the pathogenesis of SCZ. Some studies point 
to abnormalities in glutamate transmission as a cause of SCZ. This hypothesis proposes that an impairment or hypofunction of NMDAR would cause an excessive glutamate release (for review see Adell, Jiménez-Sánchez, López-Gil, \& Romón, 2012; Moghaddam \& Javitt, 2012). More specifically, a reduction in activity of NMDAR on inhibitory neurons would lead to the disinhibition of glutamate neurons, further increasing presynaptic glutamate release. Indeed, alterations in the activation of intracellular postsynaptic NMDAR signalling effectors associated with NMDAR hypoactivity have been described in postmortem PFC of SCZ patients, which may be related to the pathogenesis of the disease (Banerjee et al., 2015). In support of this hypothesis, the use of NMDAR antagonists induces schizophrenic-like symptoms in healthy individuals. For instance, resting-state functional connectivity experiments showed that the non-competitive NMDAR antagonist ketamine produces alterations in functional connectivity in different brain areas similar to those observed in patients with schizophrenia (Fleming et al., 2019; Mueller et al., 2018). These data suggest that disturbances in NMDAR activation or inhibition of NMDAR-mediated transmission may be implicated in the induction of schizophrenic symptoms. As the activation of NMDARs requires the binding of both glutamate and the co-agonists D-serine or glycine to their sites of action, astrocytes may play an important role in the etiopathogenesis of SCZ because of their control on the release of D-serine and/or glycine, especially relevant in pathological states (Ivanov \& Mothet, 2018; Papouin \& Oliet, 2014). Indeed, levels of D-serine and serine racemase (SR), the enzyme responsible for D-serine biosynthesis, have been shown to be reduced in the cerebrospinal fluid of SCZ patients (Bendikov et al., 2007). Conversely, the activity of the D-serine degradation enzyme D-amino acid oxidase (DAAO) has been found to be twofold higher in the SCZ patients than in healthy controls, further supporting the relevance of astrocytes in SCZ disorder (Madeira, Freitas, Vargas-Lopes, Wolosker, \& Panizzutti, 2008). Studies in rodents which display schizophrenia-related behavioural deficits have further reported that the genetic inactivation of DAAO enhanced D-serine levels in the brain and rescued behavioural deficits associated with SCZ-like symptoms (Labrie, Wang, Barger, Baker, \& Roder, 2010). Other groups also reported about a genetic deletion of SR which was associated with a reduction in dendritic spine density and hippocampal volume and an impairment in hippocampal plasticity and cognitive abnormalities, further supporting a role of D-serine and associated enzymes in the pathogenesis of SCZ. Moreover, direct treatment of animals with D-serine at adult stages reversed the electrophysiological, neurochemical and cognitive deficits of SCZ (Balu et al., 2013). Furthermore, direct supplementation of juvenile and adolescent mice with D-serine prevented the onset of SCZ cognitive-like deficits in adult offspring of female mice with induced maternal immune activation by viral mimetic activation with polyinosinic:polycytidylic acid (known as poly(I:C)) (Fujita, Ishima, \& Hashimoto, 2016).

Studies performed with iPSCs additionally revealed that SCZ patient-derived neurons showed alterations in neurite outgrowth, in PSD-95/MAP2AB ratio levels and in the expression of glutamate receptors (Brennand et al., 2011). More specifically, patient-derived glutamatergic neurons displayed significantly less staining of the pre- and postsynaptic markers synapsin 1 and PSD-95, respectively, with associated reduced ability to form synaptic contacts (Robicsek et al., 2013).

Among further studies, a genome-wide association study (GWAS) has shown genetic evidence for the role of glia cells in SCZ. Astrocyte- and oligodendrocyte-specific genes, but not microglial-specific ones, are associated with an enhanced risk to develop schizophrenia. Among the astrocytic genes which came up in that study, especially remarkable were those involved in functions at the tripartite synapse and in signal transduction, such as genes implicated in intracellular astrocytic responses such as calcium elevations or gap junction coupling, astrocyte-mediated regulation of neurotransmitter balances via neurotransmitter transporters or release of gliotransmitters, and/or astrocytic modulation of synapse strength and synapse morphology or involved in cell-matrix interactions (Goudriaan et al., 2014). Moreover, in human DNA samples from SCZ patients, single-nucleotide polymorphisms in genes that play a role in phagocytic pathways have been identified, further linking phagocytosis to the disease state. Evidences from both linkage association studies and expression studies, using postmortem brain cDNA libraries, suggest that MEGF10 may be associated with SCZ (Chen et al., 2008). Moreover, the reduced activity of glutamatergic synapses may further involve synaptic phagocytic mechanisms in the onset/development of SCZ because of the importance that the activity-dependent astrocyte-mediated refinement of neuronal circuits plays during the early stages of brain postnatal development, which may not properly occur in SCZ brains. Apart from MEGF10, genetic evidence suggests that GULP1, the adapter protein required for efficient phagocytosis, is altered in SCZ. As these genes are necessary to activate the process of phagocytosis, their impairment may be related to the formation of abnormal synaptic connections and lead to disease progression (Chen et al., 2009).

\section{5 | PUTATIVE INVOLVEMENT OF GLIAL CELLS IN THE E/I BALANCE REGULATION AND IMPLICATIONS FOR PSYCHIATRIC DISORDERS}

A hypothetical role for an E/I imbalance in neuropsychiatric disorders has been mostly proposed and defined for ASD (Gao \& Penzes, 2015; Rubenstein \& Merzenich, 
2003; Sohal \& Rubenstein, 2019). This hypothesis stemmed from the observation that many gene mutations associated with ASD are linked to the proper development of the glutamatergic and GABAergic neurotransmitter systems, thereby suggesting that imbalances in their respective synaptic properties, with a focus on number and strength of excitatory and inhibitory synapses, might underlie the etiopathogenesis of ASD. In view of similar findings in other psychopathologies, it is conceivable to consider this "E/I imbalance hypothesis" of brain disorders as a common thread line linking several neuropsychiatric disorders. In addition, given that various homoeostatic mechanisms, including forms of plasticity and refinement of circuits, occur during the early stages of postnatal development, the critical period should be considered as a target for future studies and treatment options. Although neurons and especially synapses seem to be the worst affected structures in neurodevelopmental and neuropsychiatric disorders, a large body of evidence is now putting a high relevance on the involvement of glial cells in such disorders, especially because of the emerging key roles played by these cells in the shaping of neuronal networks and functions.

We already mentioned that astrocytes play a critical role in the activity-dependent selective synapse elimination which favours the remodelling of excitatory or inhibitory inputs (Chung et al., 2013). The close proximity of astrocyte processes to the synapses renders these cells ideal candidates for controlling and eventually restoring E/I balance in the brain. On the one hand, astrocytes exert their regulatory functions on glutamatergic and GABAergic neurotransmission. On the other hand, astrocytes directly reshape neuronal circuits through their phagocytic activity directly on synapses.

For the modulation of glutamatergic and GABAergic neurotransmission, specific glutamate receptors and transporters found in astrocytes are activated upon glutamate release at the synapse. They protect the brain from glutamate-induced overexcitation of neurons and excitotoxicity and control the concentration of glutamate in the synaptic cleft (for review, see Parpura et al., 2012; Schousboe, Scafidi, Bak, Waagepetersen, \& McKenna, 2014). Astrocytes also express GABA receptors and transporters and respond to extracellular GABA. Interestingly, ex vivo experiments show that astrocyte reactivity driven by viral induction leads to a selective reduction of inhibitory currents as a consequence of rapid GABA depletion induced by downregulation of the astrocytic glutamine synthetase enzyme (Ortinski et al., 2010). Because reactive astrocytes may additionally become a major source of D-serine in pathological conditions (Ivanov \& Mothet, 2018) and may thereby over-activate NMDAR, we might hypothesize that such events also lead to an E/I imbalance, with consequent onset of brain disorders. Indeed, a reduced inhibitory function has been, for example, described in MDD, characterized by a reduced concentration of the inhibitory GABA neurotransmitter and alterations in the subunit composition of GABAergic receptors which affected their activity (for review see Luscher, Shen, \& Sahir, 2011). Additionally, changes in the inhibitory system have also been reported in ASD. Studies of autistic brains revealed reduced expression of glutamic acid decarboxylase (GAD) 65 and GAD67, the major enzymes that modulate GABA synthesis from L-glutamate (Blatt \& Fatemi, 2011). Moreover, various GABA receptor subunits were found to be reduced in superior frontal cortex, parietal cortex and cerebellum of postmortem brains from patients (Blatt \& Fatemi, 2011). Markers of inhibitory neurotransmission are altered in the PFC of subjects with SCZ, with reported selective reductions of specific interneuron gene expression, such as somatostatin and neuropeptide Y mRNAs (Fung, Fillman, Webster, \& Shannon, 2014). Apart from changes in synaptic modulation, a reduction in the expression of synapse-related genes and changes in the number of spine densities have been found in neuropsychiatric disorders using microarray gene profiling and electron microscopic stereology (Kang et al., 2012). Among such genes, a few are classified as regulators of synaptic vesicles, such as calmodulin 2 and synapsin, and regulators of dendritic spine formation, such as Rab3A and Rab4B. Moreover, mediators of axonal outgrowth and regeneration, such as beta-tubulin 4 , are known to be decreased in MDD subjects. A decrease in the expression of these genes in the PFC is exhibited in rodent models of chronic unpredictable stress as well (Kang et al., 2012). In postmortem studies of ASD, an increased dendritic spine density with reduced spine pruning in pyramidal neurons of layer $\mathrm{V}$ of the cortex has been reported during development. Similar results have been seen in $\mathrm{Tsc}^{+/-}$animal model of autism (Tang et al., 2014), further suggesting a dysregulation of synaptic pruning in neuropsychiatric disorders. Neuropathological studies in patients with SCZ revealed alterations in synaptic densities in prefrontal cortical projection neurons. Particularly interesting is the described reduction in synaptic densities (Konopaske, Lange, Coyle, \& Benes, 2014; MacDonald et al., 2017) and downregulation of expression levels of mRNAs encoding for proteins known to be key regulators of dendritic spine formation (Hill, Hashimoto, \& Lewis, 2006). These synapse-related deficits appear to affect multiple cortical and subcortical regions, involving both glutamatergic projection neurons and multiple classes of GABAergic inhibitory interneurons (Hill et al., 2006; Nakazawa et al., 2011). Synaptic activity is the strongest trigger of phagocytic processes, thus allowing us to propose that excitatory/inhibitory synaptic activity can be affected by glia-mediated synapse engulfment, highlighting a possible involvement of astrocytes as a cause of $\mathrm{E} / \mathrm{I}$ imbalance in brain pathologies. An altered excitatory and inhibitory activity, which takes place already since developmental stages, may lead to subsequent synaptic dysfunction(s) in specific networks. This could in turn affect signal processing, thereby leading to the phenotypic changes observed in the various psychiatric disorders (Figure 1). 


\section{(a) Physiological conditions}

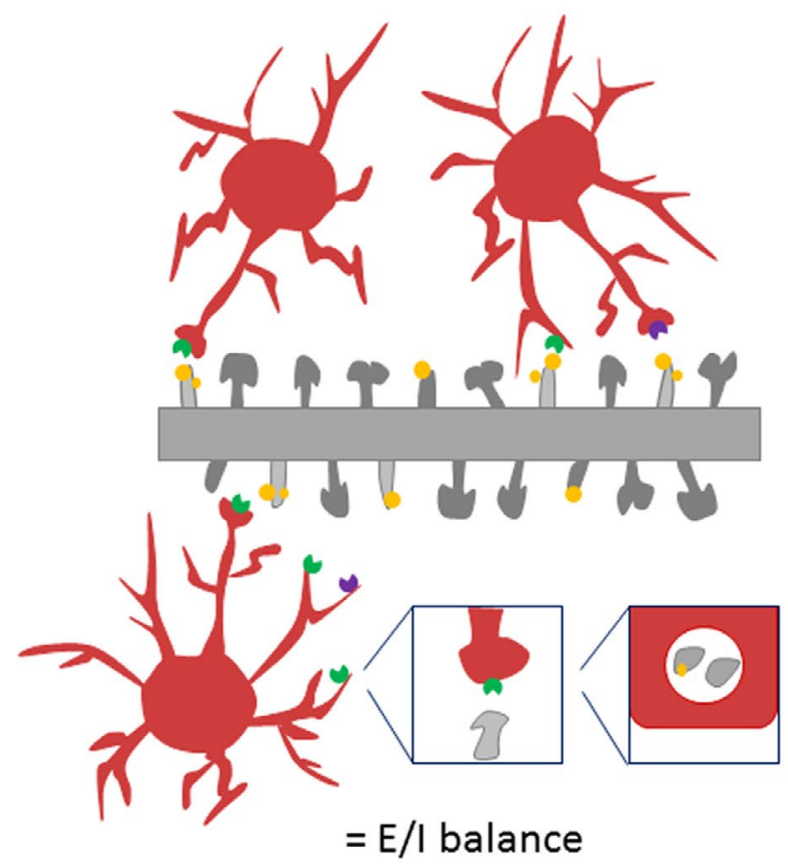

(b) Psychiatric disorders

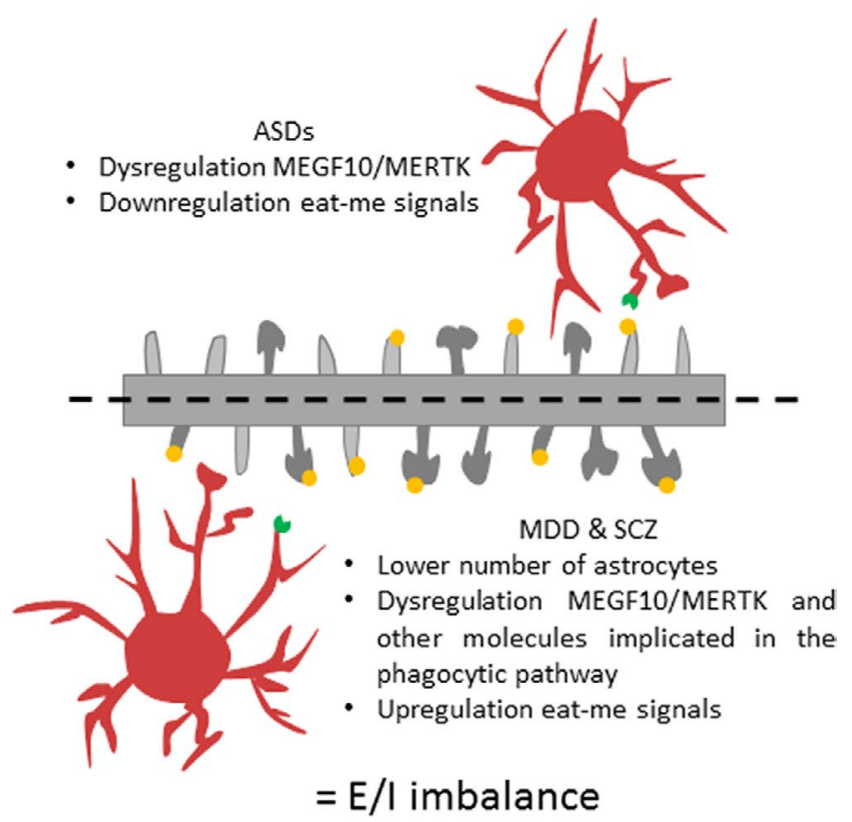

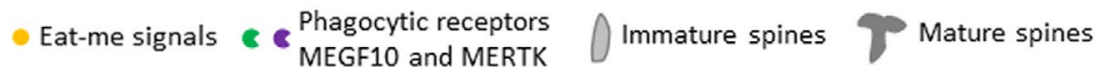

F I G U RE 1 Astrocytes control synapse elimination. (a) In healthy conditions, astrocytes mediate synapse elimination in order to refine neuronal circuits. Astrocytes mediate pruning during development and in the adult nervous system through the activation of the MEGF10 and MERTK phagocytic pathways, thereby contributing to the excitatory/inhibitory (E/I) balance. (b) In psychiatric disorders, molecules involved in the phagocytic pathway may be dysregulated since developmental stages, giving rise to an improper refinement of circuits. In major depressive disorder, a lower number of astrocytes may be cause of a declining rate of synapse engulfment contributing to an E/I imbalance. Furthermore, a downregulation of "eat-me" signals may be responsible for an increase in spine densities with immature morphology, as found in autism spectrum disorders. In schizophrenia, instead, an upregulation of "eat-me" signals may be responsible for an excessive pruning activity, which leads to a reduced amount of excitatory synapses. Those activities may take place during the critical period, what could explain the early onset of some psychiatric disorders. ASD, autism spectrum disorder; MDD, major depressive disorder; MEGF10, Multiple EGF Like Domains 10; MERTK, proto-oncogene tyrosine-protein kinase; SCZ, schizophrenia

Next step of investigations should support a deeper understanding on the process of synapse remodelling mediated by glia cells. A more refined knowledge about how neuronal activity controls the rate of astrocyte-mediated synapse phagocytosis, for instance based on the modulation of "eat-me" signals in physiological conditions, may help to formulate innovative hypothesis about what may occur in pathological conditions during developmental stages and/or in adulthood. Understanding whether glial cells are the initial causes of E/I imbalances will allow us to target those cells as an effective therapy to revert an improper synaptic pruning in psychiatric disorders.

\section{6 | CONCLUSIONS AND PERSPECTIVES}

Defects in synapse elimination which may result in unwanted synapse loss or may give rise to supernumerary synapses might contribute to pathophysiological features shared by many neuropsychiatric diseases (Bian, Miao, He, Qiu, \& Yu, 2015; Tang et al., 2014). In the last decade, growing evidences have shown the impact of glial cells on synapse elimination (Chung et al., 2013; Schafer et al., 2012; Stevens et al., 2007). As a consequence, both excitatory and inhibitory neuronal activities can be affected when glia cells do not perform proper synaptic pruning. The reduced number and impaired morphology of astrocytes described in brains from MDD patients, which might be true for other psychopathologies, could lead to a declining rate of synapse engulfment during the critical period. This may result in imbalanced neurotransmission and subsequent synaptic dysfunction, contributing to a disease phenotype that may represent a common underlying mechanism behind several psychiatric disorders. Further studies are necessary to reveal if changes in the rate of pruning during development could contribute to changes in the critical period plasticity, triggering the synaptic dysfunctions. 


\section{ACKNOWLEDGEMENTS}

This work was supported by intramural funding from the Department of Psychiatry and Psychotherapy of the University of Regensburg and by the German Research Council (DFG-GRK2174 "Neurobiology of Emotion Dysfunction" (P1)) to BDB. The sponsors did not have any role in writing the report and in the decision to submit the article for publication.

\section{CONFLICT OF INTEREST}

The authors declare that the research was conducted in the absence of any commercial or financial relationships that could be construed as a potential competing interest.

\section{AUTHOR CONTRIBUTION}

$\mathrm{CR}$ and LE contributed to literature screening and drafting the manuscript. BDB contributed to editing and revising the final version of the manuscript. All authors read and approved the final version of the manuscript.

\section{PEER REVIEW}

The peer review history for this article is available at https:// publons.com/publon/10.1111/ejn.14900.

\section{ORCID}

Barbara Di Benedetto (D) https://orcid.
org/0000-0001-6537-9072

\section{REFERENCES}

Adell, A., Jiménez-Sánchez, L., López-Gil, X., \& Romón, T. (2012). Is the acute NMDA receptor hypofunction a valid model of schizophrenia. Schizophrenia Bulletin, 38(1), 9-14.

Allen, N. J., Bennett, M. L., Foo, L. C., Wang, G. X., Chakraborty, C., Smith, S. J., \& Barres, B. A. (2012). Astrocyte glypicans 4 and 6 promote formation of excitatory synapses via GluA1 AMPA receptors. Nature, 486(7403), 410-414.

Anderson, H. A., Maylock, C. A., Williams, J. A., Paweletz, C. P., Shu, H., \& Shacter, E. (2003). Serum-derived protein S binds to phosphatidylserine and stimulates the phagocytosis of apoptotic cells. Nature Immunology, 4(1), 87-91.

Araque, A., Parpura, V., Sanzgiri, R. P., \& Haydon, P. G. (1999). Tripartite synapses: Glia, the unacknowledged partner. Trends in Neurosciences, 22(5), 208-215.

Balu, D. T., Li, Y., Puhl, M. D., Benneyworth, M. A., Basu, A. C., Takagi, S., ... Coyle, J. T. (2013). Multiple risk pathways for schizophrenia converge in serine racemase knockout mice, a mouse model of NMDA receptor hypofunction. Proceedings of the National Academy of Sciences of the United States of America, 110(26), E2400-9.

Banasr, M., Dwyer, J. M., \& Duman, R. S. (2011). Cell atrophy and loss in depression: Reversal by antidepressant treatment. Current Opinion in Cell Biology, 23(6), 730-737.

Banerjee, A., Wang, H. Y., Borgmann-Winter, K. E., MacDonald, M. L., Kaprielian, H., Stucky, A., ... Hahn, C. G. (2015). Src kinase as a mediator of convergent molecular abnormalities leading to
NMDAR hypoactivity in schizophrenia. Molecular Psychiatry, 20(9), 1091-1100.

Barker, A. J., Koch, S. M., Reed, J., Barres, B. A., \& Ullian, E. M. (2008). Developmental control of synaptic receptivity. Journal of Neuroscience, 28(33), 8150-8160.

Barker, A. J., \& Ullian, E. M. (2008). New roles for astrocytes in developing synaptic circuits. Communicative \& Integrative Biology, 1(2), 207-211.

Bejjani, A., O`Neill, J., Kim, J. A., Frew, A. J., Yee, V. W., Ly, R., ... Levitt, J. G. (2012). Elevated glutamatergic compounds in pregenual anterior cingulate in pediatric autism spectrum disorder demonstrated by 1H MRS and 1H MRSI. PLoS One, 7(7), e38786.

Bendikov, I., Nadri, C., Amar, S., Panizzutti, R., De Miranda, J., Wolosker, H., \& Agam, G. (2007). A CSF and postmortem brain study of d-serine metabolic parameters in schizophrenia. Schizophrenia Research, 90(1-3), 41-51.

Bernard, R., Kerman, I. A., Thompson, R. C., Jones, E. G., Bunney, W. E., Barchas, J. D., ... Watson, S. J. (2011). Altered expression of glutamate signaling, growth factor, and glia genes in the locus coeruleus of patients with major depression. Molecular Psychiatry, 16(6), 634-646.

Bialas, A. R., \& Stevens, B. (2013). TGF- $\beta$ signaling regulates neuronal C1q expression and developmental synaptic refinement. Nature Neuroscience, 16(12), 1773-1782.

Bian, W. J., Miao, W. Y., He, S. J., Qiu, Z., \& Yu, X. (2015). Coordinated spine pruning and maturation mediated by inter-spine competition for cadherin/catenin complexes. Cell, 162(4), 808-822.

Blatt, G. J., \& Fatemi, S. H. (2011). Alterations in GABAergic biomarkers in the autism brain: Research findings and clinical implications. Anatomical Record (Hoboken), 294(10), 1646-1652.

Brennand, K. J., Simone, A., Jou, J., Gelboin-Burkhart, C., Tran, N., Sangar, S., ... Gage, F. H. (2011). Modelling schizophrenia using human induced pluripotent stem cells. Nature, 473(7346), 221-225.

Cahoy, J. D., Emery, B., Kaushal, A., Foo, L. C., Zamanian, J. L., Christopherson, K. S., ... Barres, B. A. (2008). A transcriptome database for astrocytes, neurons, and oligodendrocytes: A new resource for understanding brain development and function. The Journal of Neuroscience, 28(1), 264-278.

Chen, X., Sun, C., Chen, Q., O’Neill, F. A., Walsh, D., Fanous, A. H., ... Kendler, K. S. (2009). Apoptotic engulfment pathway and schizophrenia. PLoS One, 4(9), e6875.

Chen, X., Wang, X., Chen, Q., Williamson, V., van den Oord, E., Maher, B. S., ... Kendler, K. S. (2008). MEGF10 Association with Schizophrenia. Biological Psychiatry, 63(5), 441-448.

Choudary, P. V., Molnar, M., Evans, S. J., Tomita, H., Li, J. Z., Vawter, M. P., ... Jones, E. G. (2005). Altered cortical glutamatergic and GABAergic signal transmission with glial involvement in depression. Proceedings of the National Academy of Sciences of the United States of America, 102(43), 15653-15658.

Christian, K. M., Miracle, A. D., Wellman, C. L., \& Nakazawa, K. (2011). Chronic stress-induced hippocampal dendritic retraction requires CA3 NMDA receptors. Neuroscience, 174, 26-36.

Chu, Y., Jin, X., Parada, I., Pesic, A., Stevens, B., Barres, B., \& Prince, D. A. (2010). Enhanced synaptic connectivity and epilepsy in C1q knockout mice. Proceedings of the National Academy of Sciences of the United States of America, 107(17), 7975-7980.

Chung, W. S., Allen, N. J., \& Eroglu, C. (2015). Astrocytes control synapse formation, Function, and elimination. Cold Spring Harbor Perspectives in Biology, 7(9), 1-18. 
Chung, W. S., Clarke, L. E., Wang, G. X., Stafford, B. K., Sher, A., Chakraborty, C., ... Barres, B. A. (2013). Astrocytes mediate synapse elimination through MEGF10 and MERTK pathways. Nature, 504(7480), 394-400.

Chung, W. S., Welsh, C. A., Barres, B. A., \& Stevens, B. (2015). The interplay between neurons and glia in synapse development and plasticity. Nature Neuroscience, 18(11), 1539-1545.

Clarke, L. E., \& Barres, B. A. (2013). Emerging roles of astrocytes in neural circuit development. Nature Reviews Neuroscience, 14(5), 311-321.

Crawford, D. C., Jiang, X., Taylor, A., \& Mennerick, S. (2012). Astrocytederived thrombospondins mediate the development of hippocampal presynaptic plasticity in vitro. Journal of Neuroscience, 32(38), 13100-13110.

Csabai, D., Wiborg, O., \& Czéh, B. (2018). Reduced synapse and axon numbers in the prefrontal cortex of rats subjected to a chronic stress model for depression. Frontiers in Cellular Neuroscience, 30(12), 24.

Czéh, B., \& Di Benedetto, B. (2013). Antidepressants act directly on astrocytes: Evidences and functional consequences. European Neuropsychopharmacology, 23(3), 171-185.

Di Benedetto, B., Malik, V. A., Begum, S., Jablonowski, L., GómezGonzález, G. B., Neumann, I. D., \& Rupprecht, R. (2016). Fluoxetine requires the endfeet protein aquaporin-4 to enhance plasticity of astrocyte processes. Frontiers in Cellular Neuroscience, 2(10), 8-14.

Di Benedetto, B., \& Rupprecht, R. (2013). Targeting glia cells: Novel perspectives for the treatment of neuropsychiatric diseases. Current Neuropharmacology, 11(2), 171-185.

Di Benedetto, B., Rupprecht, R., \& Czéh, B. (2013). Talking to the synapse: How antidepressants can target glial cells to reshape brain circuits. Current Drug Targets, 14(11), 1329-1335.

Duman, R. S., Aghajanian, G. K., Sanacora, G., \& Krystal, J. H. (2016). Synaptic plasticity and depression: New insights from stress and rapid-acting antidepressants. Nature Medicine, 22(3), 238-249.

Duman, R. S., \& Li, N. (2012). A neurotrophic hypothesis of depression: Role of synaptogenesis in the actions of NMDA receptor antagonists. Philosophical Transactions of the Royal Society of London. Series B, Biological Sciences, 367(1601), 2475-2484.

Elmariah, S. B., Hughes, E. G., Oh, E. J., \& Balice-Gordon, R. (2004). Neurotrophin signaling among neurons and glia during formation of tripartite synapses. Neuron Glia Biology, 1(4), 1-11.

Emberti Gialloreti, L., \& Curatolo, P. (2018). Autism spectrum disorder: Why do we know so little? Frontiers in Neurology, 9, 670.

Ernst, C., Nagy, C., Kim, S., Yang, J. P., Deng, X., Hellstrom, I. C., ... Turecki, G. (2011). Dysfunction of astrocyte connexins 30 and 43 in dorsal lateral prefrontal cortex of suicide completers. Biological Psychiatry, 70(4), 312-319.

Farhy-Tselnicker, I., \& Allen, N. J. (2018). Astrocytes, neurons, synapses: A tripartite view on cortical circuit development. Neural Development, 13(1), 7.

Fatemi, S. H., Folsom, T. D., Reutiman, T. J., \& Lee, S. (2008). Expression of astrocytic markers aquaporin 4 and connexin 43 is altered in brains of subjects with autism. Synapse (New York, N. Y.), 62(7), 501-507.

Fleming, L. M., Javitt, D. C., Carter, C. S., Kantrowitz, J. T., Girgis, R. R., Kegeles, L. S., ... Corlett, P. R. (2019). A multicenter study of ketamine effects on functional connectivity: Large scale network relationships, hubs and symptom mechanisms. NeuroImage Clinical, 22, 101739 .
Fujita, Y., Ishima, T., \& Hashimoto, K. (2016). Supplementation with D-serine prevents the onset of cognitive deficits in adult offspring after maternal immune activation. Scientific Reports, 6, 37261.

Fullard, J. F., \& Baker, N. E. (2015). Signaling by the engulfment receptor draper: A screen in drosophila melanogaster implicates cytoskeletal regulators, Jun N-Terminal Kinase, and Yorkie. Genetics, 199(1), 117-134.

Fung, S. J., Fillman, S. G., Webster, M. J., \& Shannon, W. C. (2014). Schizophrenia and bipolar disorder show both common and distinct changes in cortical interneuron markers. Schizophrenia Research, 155(1-3), 26-30.

Gao, R., \& Penzes, P. (2015). Common mechanisms of excitatory and inhibitory imbalance in schizophrenia and autism spectrum disorders. Current Molecular Medicine, 15, 146-167.

Goritz, C., Mauch, D. H., \& Pfrieger, F. W. (2005). Multiple mechanisms mediate cholesterol-induced synaptogenesis in a CNS neuron. Molecular and Cellular Neuroscience, 29(2), 190-201.

Goudriaan, A., De Leeuw, C., Ripke, S., Hultman, C. M., Sklar, P., Sullivan, P. F., ... Verheijen, M. H. (2014). Specific glial functions contribute to Schizophrenia susceptibility. Schizophrenia Bulletin, 40(4), 925-935.

Hajszan, T., Dow, A., Warner-Schmidt, J. L., Szigeti-Buck, K., Sallam, N. L., Parducz, A., ... Duman, R. S. (2009). Remodeling of hippocampal spine synapses in the rat learned helplessness model of depression. Biological Psychiatry, 65(5), 392-400.

Hama, H., Hara, C., Yamaguchi, K., \& Miyawaki, A. (2004). PKC signaling mediates global enhancement of excitatory synaptogenesis in neurons triggered by local contact with astrocytes. Neuron, 41(3), 405-415.

Hamed, N. O., Al-Ayadhi, L., Osman, M. A., Elkhawad, A. O., Qasem, H., Al-Marshoud, M., ... El-Ansary, A. (2018). Understanding the roles of glutamine synthetase, glutaminase, and glutamate decarboxylase autoantibodies in imbalanced excitatory/inhibitory neurotransmission as etiological mechanisms of autism. Psychiatry and Clinical Neurosciences, 72(5), 362-373.

Hamon, Y., Trompier, D., Ma, Z., Venegas, V., Pophillat, M., Mignotte, V., ... Chimini, G. (2006). Cooperation between engulfment receptors: The case of ABCA1 and MEGF10. PLoS One, 1(1), e120.

Henneberger, C., Papouin, T., Oliet, S., \& Rusakov, D. A. (2010). Longterm potentiation depends on release of d-serine from astrocytes. Nature, 463, 232-236.

Hill, J. J., Hashimoto, T., \& Lewis, D. A. (2006). Molecular mechanisms contributing to dendritic spine alterations in the prefrontal cortex of subjects with schizophrenia. Molecular Psychiatry, 11(6), 557-566.

Holmes, S. E., Scheinost, D., Finnema, S. J., Naganawa, M., Davis, M. T., DellaGioia, N., ... Esterlis, I. (2019). Lower synaptic density is associated with depression severity and network alterations. Nature Communications, 10(1), 1529.

Hughes, E. G., Elmariah, S. B., \& Balice-Gordon, R. J. (2010). Astrocyte secreted proteins selectively increase hippocampal GABAergic axon length, branching, and synaptogenesis. Molecular and Cellular Neuroscience, 43(1), 136-145.

Iram, T., Ramirez-Ortiz, Z., Byrne, M. H., Coleman, U. A., Kingery, N. D., Means, T. K., ... El Khoury, J. (2016). Megf10 is a receptor for $\mathrm{C} 1 \mathrm{Q}$ that mediates clearance of apoptotic cells by astrocytes. Journal of Neuroscience, 36(19), 5185-5192.

Ivanov, A. D., \& Mothet, J. P. (2018). The plastic D-serine signaling pathway: Sliding from neurons to glia and vice-versa. Neuroscience Letters, 689, 21-25. 
Kang, H. J., Voleti, B., Hajszan, T., Rajkowska, G., Stockmeier, C. A., Licznerski, P., ... Duman, R. S. (2012). Decreased expression of synapse-related genes and loss of synapses in major depressive disorder. Nature Medicine, 18(9), 1413-1417.

Kong, H., Zeng, X. N., Fan, Y., Yuan, S. T., Ge, S., Xie, W. P. et al (2014). Aquaporin-4 knockout exacerbates corticosterone-induced depression by inhibiting astrocyte function and hippocampal neurogenesis. CNS Neuroscience \& Therapeutics, 20, 391-402.

Konopaske, G. T., Lange, N., Coyle, J. T., \& Benes, F. M. (2014). Prefrontal cortical dendritic spine pathology in schizophrenia and bipolar disorder. JAMA Psychiatry, 71(12), 1323-1331.

Kucukdereli, H., Allen, N. J., Lee, A. T., Feng, A., Ozlu, M. I., Conatser, L. M., ... Eroglu, C. (2011). Control of excitatory CNS synaptogenesis by astrocyte-secreted proteins Hevin and SPARC. Proceedings of the National Academy of Sciences of the United States of America, 108(32), E440-E449.

Labrie, V., Wang, W., Barger, S. W., Baker, G. B., \& Roder, J. C. (2010). Genetic loss of D-amino acid oxidase activity reverses schizophrenia-like phenotypes in mice. Genes, Brain and Behavior, 9(1), $11-25$.

Lee, E., \& Chung, W. S. (2019). Glial control of synapse number in healthy and diseased brain. Frontiers in Cellular Neuroscience, 13, 42.

Liu, R.-J., \& Aghajanian, G. K. (2008). Stress blunts serotonin- and hypocretin-evoked EPSCs in prefrontal cortex: Role of corticosterone-mediated apical dendritic atrophy. Proceedings of the National Academy of Sciences of the United States of America, 105(1), 359-364.

Luchena, C., Zuazo-Ibarra, J., Alberdi, E., Matute, C., \& CapetilloZarate, E. (2018). Contribution of neurons and glial cells to complement-mediated synapse removal during development, aging and in Alzheimer's disease. Mediators of Inflammation, 18, 2530414.

Luscher, B., Shen, Q., \& Sahir, N. (2011). The GABAergic deficit hypothesis of major depressive disorder. Molecular Psychiatry, 16(4), 383-406.

MacDonald, J. M., Beach, M. G., Porpiglia, E., Sheehan, A. E., Watts, R. J., \& Freeman, M. R. (2006). The drosophila cell corpse engulfment receptor draper mediates glial clearance of severed axons. Neuron, 50(6), 869-881.

MacDonald, M. L., Alhassan, J., Newman, J. T., Richard, M., Gu, H., Kelly, R. M., ... Sweet, R. A. (2017). Selective loss of smaller spines in schizophrenia. The American Journal of Psychiatry, 174(6), 586-594.

Madeira, C., Freitas, M. E., Vargas-Lopes, C., Wolosker, H., \& Panizzutti, R. (2008). Increased brain d-amino acid oxidase (DAAO) activity in schizophrenia. Schizophrenia Research, 101(1-3), 76-83.

Mao, Y., \& Finnemann, S. C. (2015). Regulation of phagocytosis by Rho GTPases. Small GTPases, 6(2), 89-99.

Mapes, J., Chen, Y. Z., Kim, A., Mitani, S., Kang, B. H., \& Xue, D. (2012). CED-1, CED-7, and TTR-52 regulate surface phosphatidylserine expression on apoptotic and phagocytic cells. Current Biology, 22(14), 1267-1275.

Maras, P. M., Molet, J., Chen, Y., Rice, C., Ji, S. G., Solodkin, A., \& Baram, T. Z. (2014). Preferential loss of dorsal-hippocampus synapses underlies memory impairments provoked by short, multimodal stress. Molecular Psychiatry, 19(7), 811-822.

Marder, S. R., \& Cannon, T. D. (2019). Schizophrenia. New England Journal of Medicine, 381(18), 1753-1761.
Mauch, D. H., Nägier, K., Schumacher, S., Göritz, C., Müller, E. C., Otto, A., \& Pfrieger, F. W. (2001). CNS synaptogenesis promoted by glia-derived cholesterol. Science, 294(5545), 1354-1357.

Mayorquin, L. C., Rodriguez, A. V., Sutachan, J. J., \& Albarracín, S. L. (2018). Connexin-mediated functional and metabolic coupling between astrocytes and neurons. Frontiers in Molecular Neuroscience, $11,118$.

Medina, A., Watson, S. J., Bunney, W., Myers, R. M., Schatzberg, A., Barchas, J., ... Thompson, R. C. (2016). Evidence for alterations of the glial syncytial function in major depressive disorder. Journal of Psychiatric Research, 72, 15-21.

Meunier, C. N., Dallérac, G., Le Roux, N., Sacchi, S., Levasseur, G., Amar, M., ... Fossier, P. (2016). D-serine and glycine differentially control neurotransmission during visual cortex critical period. PLoS One, 11(3), e0151233.

Miguel-Hidalgo, J. J., Waltzer, R., Whittom, A. A., Austin, M. C., Rajkowska, G., \& Stockmeier, C. A. (2010). Glial and glutamatergic markers in depression, alcoholism, and their comorbidity. Journal of Affective Disorders, 127(1-3), 230-240.

Moghaddam, B., \& Javitt, D. (2012). From revolution to evolution: The glutamate hypothesis of schizophrenia and its implication for treatment. Neuropsychopharmacology, 37(1), 4-15.

Mueller, F., Musso, F., London, M., de Boer, P., Zacharias, N., \& Winterer, G. (2018). Pharmacological fMRI: Effects of subanesthetic ketamine on resting-state functional connectivity in the default mode network, salience network, dorsal attention network and executive control network. NeuroImage Clinical, 19, 745-757.

Murakoshi, H., \& Yasuda, R. (2012). Postsynaptic signaling during plasticity of dendritic spines. Trends in Neurosciences, 35(2), 135-143.

Murphy-Royal, C., Johnston, A. D., Boyce, A. K. J., Diaz-Castro, B., Institoris, A., Peringod, G., ... Gordon G. R. (2020). Stress gates an astrocytic energy reservoir to impair synaptic plasticity. Nature Communications, 11(1). https://doi.org/10.1038/s41467-020-15778-9

Nakazawa, K., Zsiros, V., Jiang, Z., Nakao, K., Kolata, S., Zhang, S., \& Belforte, J. E. (2011). GABAergic interneuron origin of schizophrenia pathophysiology. Neuropharmacology, 62(3), 1574-1583.

Ortinski, P. I., Dong, J., Mungenast, A., Yue, C., Takano, H., Watson, D. J., ... Coulter, D. A. (2010). Selective induction of astrocytic gliosis generates deficits in neuronal inhibition. Nature Neuroscience, 13(5), 584-591.

Otte, C., Gold, S., Penninx, B., Pariante, C. M., Etkin, A., Fava, M., ... Schatzberg, A. F. (2016). Major depressive disorder. Nature Reviews Disease Primers, 2, 16065.

Païdassi, H., Tacnet-Delorme, P., Garlatti, V., Darnault, C., Ghebrehiwet, B., Gaboriaud, C., ... Frachet, P. (2008). C1q binds phosphatidylserine and likely acts as a multiligand-bridging molecule in apoptotic Cell recognition. The Journal of Immunology, 180(4), 2329-2338.

Panatier, A., Theodosis, D. T., Mothet, J. P., Touquet, B., Pollegioni, L., Poulain, D. A., \& Oliet, S. H. R. (2006). Glia-derived d-serine controls NMDA receptor activity and synaptic memory. Cell, 125(4), 775-784.

Papouin, T., \& Oliet, S. H. R. (2014). Organization, control and function of extrasynaptic NMDA receptors. Philosophical Transactions of the Royal Society of London. Series B, Biological Sciences, 369(1654), 18-21.

Parpura, V., Heneka, M. T., Montana, V., Oliet, S. H. R., Schousboe, A., Haydon, P. G., ... Verkhratsky, A. (2012). Glial cells in (patho) physiology. Journal of Neurochemistry, 121(1), 4-27. 
Perea, G., Navarrete, M., \& Araque, A. (2009). Tripartite synapses: Astrocytes process and control synaptic information. Trends in Neurosciences, 32(8), 421-431.

Popoli, M., Yan, Z., McEwen, B. S., \& Sanacora, G. (2012). The stressed synapse: The impact of stress and glucocorticoids on glutamate transmission. Nature Reviews Neuroscience, 13(1), 22-37.

Radley, J. J., Rocher, A. B., Miller, M., Janssen, W. G., Liston, C., Hof, P. R., ... Morrison, J. H. (2006). Repeated stress induces dendritic spine loss in the rat medial prefrontal cortex. Cerebral Cortex, 16(3), 313-320.

Rajkowska, G., Hughes, J., Stockmeier, C. A., Javier Miguel-Hidalgo, J., \& Maciag, D. (2013). Coverage of blood vessels by astrocytic endfeet is reduced in major depressive disorder. Biological Psychiatry, 73(7), 613-621.

Rajkowska, G., \& Stockmeier, C. (2013). Astrocyte pathology in major depressive disorder: Insights from human postmortem brain tissue. Current Drug Targets, 14(11), 1225-1236.

Robicsek, O., Karry, R., Petit, I., Salman-Kesner, N., Müller, F. J., Klein, E., ... Ben-Shachar, D. (2013). Abnormal neuronal differentiation and mitochondrial dysfunction in hair follicle-derived induced pluripotent stem cells of schizophrenia patients. Molecular Psychiatry, 18(10), 1067-1076.

Rubenstein, J. L. R., \& Merzenich, M. M. (2003). Model of autism: Increased ratio of excitation/ inhibition in key neural systems. Genes, Brain, and Behavior, 2(5), 255-267.

Russo, F. B., Freitas, B. C., Pignatari, G. C., Fernandes, I. R., Sebat, J., Muotri, A. R., \& Beltrão-Braga, P. C. B. (2018). Modeling the interplay between neurons and astrocytes in autism using human induced pluripotent stem cells. Biological Psychiatry, 83(7), 569-578.

Schafer, D. P., Lehrman, E. K., Kautzman, A. G., Koyama, R., Mardinly, A. R., Yamasaki, R., ... Stevens, B. (2012). Microglia sculpt postnatal neural circuits in an activity and complement-dependent manner. Neuron, 74(4), 691-705.

Schmidtner, A. K., Slattery, D. A., Gläsner, J., Hiergeist, A., Gryksa, K., Malik, V. A., ... Neumann, I. D. (2019). Minocycline alters behavior, microglia and the gut microbiome in a trait-anxiety-dependent manner. Translational Psychiatry, 9(1), 223.

Schousboe, A., Scafidi, S., Bak, L. K., Waagepetersen, H. S., \& McKenna, M. M. (2014). Glutamate metabolism in the brain focusing on astrocytes. Advances in Neurobiology, 11, 13-30.

Singh, S. K., Stogsdill, J. A., Pulimood, N. S., Dingsdale, H., Kim, Y. H., Pilaz, L. J., ... Eroglu, C. (2016). Astrocytes assemble thalamocortical synapses by bridging NRX1 $\alpha$ and NL1 via Hevin. Cell, 164(1-2), 183-196.

Smith, S. E. P., Zhou, Y. D., Zhang, G., Jin, Z., Stoppel, D. C., \& Anderson, M. P. (2011). Increased gene dosage of Ube3a results in autism traits and decreased glutamate synaptic transmission in mice. Science Translational Medicine, 3(103), 103 ra97.

Snyder, J. S. (2019). Recalibrating the relevance of adult neurogenesis. Trends in Neurosciences, 42(3), 164-178.

Sohal, V. S., \& Rubenstein, J. L. R. (2019). Excitation-inhibition balance as a framework for investigating mechanisms in neuropsychiatric disorders. Molecular Psychiatry, 24(9), 1248-1257.

Stevens, B., Allen, N. J., Vazquez, L. E., Howell, G. R., Christopherson, K. S., Nouri, N., ... Barres, B. A. (2007). The classical complement cascade mediates CNS synapse elimination. Cell, 131(6), $1164-1178$.
Stogsdill, J. A., \& Eroglu, C. (2017). The interplay between neurons and glia in synapse development and plasticity. Current Opinion in Neurobiology, 42, 1-8.

Stogsdill, J. A., Ramirez, J., Liu, D., Kim, Y. H., Baldwin, K. T., Enustun, E., ... Eroglu, C. (2017). Astrocytic neuroligins control astrocyte morphogenesis and synaptogenesis. Nature, 551(7679), 192-197.

Strohschein, S., Hüttmann, K., Gabriel, S., Binder, D. K., Heinemann, U., \& Steinhäuser, C. (2011). Impact of aquaporin-4 channels on $\mathrm{K}+$ buffering and gap junction coupling in the hippocampus. Glia, 59(6), 973-980.

Sun, J.-D., Liu, Y., Yuan, Y.-H., Li, J-, \& Chen, N.-H. (2012). Gap junction dysfunction in the prefrontal cortex induces depressive-like behaviors in rats . Neuropsychopharmacology, 37(5), 1305-1320.

Tabata, H. (2015). Diverse subtypes of astrocytes and their development during corticogenesis. Frontiers in Neuroscience, 9, 114.

Tanasic, S., Mattusch, C., Wagner, E. M., Eder, M., Rupprecht, R., Rammes, G., \& DiBenedetto, B. (2016). Desipramine targets astrocytes to attenuate synaptic plasticity via modulation of the ephrinA3/EphA4 signalling. Neuropharmacology, 105, 154-163.

Tang, G., Gudsnuk, K., Kuo, S. H., Cotrina, M. L., Rosoklija, G., Sosunov, A., ... Sulzer, D. (2014). Loss of mTOR-dependent macroautophagy causes autistic-like synaptic pruning deficits. Neuron, 83(5), 1131-1143.

Thompson, S. M., Kallarackal, A. J., Kvarta, M. D., Van Dyke, A. M., LeGates, T. A., \& Cai, X. (2015). An excitatory synapse hypothesis of depression. Trends in Neurosciences, 38(5), 279-294.

Ullian, E. M., Sapperstein, S. K., Christopherson, K. S., Barres, B. A., $\&$ Barde, Y. (2001). Control of synapse number by glia neuronal and glial cell biology. Current Opinion in Neurobiology, 10(5), 642-648.

Van Horn, M. R., Strasser, A., Miraucourt, L. S., Pollegioni, L., \& Ruthazer, E. S. (2017). The gliotransmitter D-serine promotes synapse maturation and axonal stabilization in Vivo. Journal of Neuroscience, 37(26), 6277-6288.

Voglis, G., \& Tavernarakis, N. (2006). The role of synaptic ion channels in synaptic plasticity. EMBO Reports, 7(11), 1104-1110.

Wiesel, D., \& Hubel, T. (1963). Effects of visual deprivation on morphology and physiology of cells in the cats lateral geniculate body. Journal of Neurophysiology, 26, 978-993.

Wilton, D. K., Dissing-Olesen, L., \& Stevens, B. (2019). Neuron-Glia signaling in synapse elimination. Annual Review of Neuroscience, 42(1), 107-127.

Zhou, Z., Hartwieg, E., \& Horvitz, H. R. (2001). CED-1 is a transmembrane receptor that mediates cell corpse engulfment in C. elegans. Cell, 104(1), 43-56.

Ziegenfuss, J. S., Biswas, R., Avery, M. A., Hong, K., Sheehan, A. E., Yeung, Y. G., \& Stanley Freeman, M. R. (2008). Draper-dependent glial phagocytic activity is mediated by Src and Syk family kinase signalling. Nature, 453(7197), 935-939.

How to cite this article: Roman C., Egert L., \& Di Benedetto B. (2021). Astrocytic-neuronal crosstalk gets jammed: Alternative perspectives on the onset of neuropsychiatric disorders. European Journal of Neuroscience, 54(5), 5717-5729. https://doi.org/10.1111/ejn.14900 